\title{
EDUCAÇÃO E INFORMAÇÃO EM SAÚDE: INICIATIVAS DOS NÚCLEOS DE TELESSAÚdE PARA O ENFRENTAMENTO DA COVID-19
}

\section{HEALTH EDUCATION AND INFORMATION: INITIATIVES OF TELEHEALTH CENTERS TO COMBAT COVID-19}

\section{EDUCACIÓN E INFORMACIÓN SANITARIA: INICIATIVAS DE CENTROS DE TELESALUD PARA HACER FRENTE AL COVID-19}

Rosangela Caetano ${ }^{1}$, Angélica Baptista Silva ${ }^{2}$, Rondineli Mendes da Silva ${ }^{3}$, Carla Cardi Nepomuceno de Paiva ${ }^{4}$, Ana Cristina Carneiro Menezes Guedes ${ }^{5}$, Gizele da Rocha Ribeiro ${ }^{6}$, Daniela Lacerda Santos ${ }^{7}$, Vanessa de Lima e Souza ${ }^{8}$, lone Ayala Gualandi de Oliveira ${ }^{9}$

\section{RESUMO}

Objetivo: Identificar as iniciativas de teleducação e informações em saúde direcionadas ao enfrentamento da pandemia da COVID-19 oferecidas pelos Núcleos Estaduais de Telessaúde vinculados ao Programa Telessaúde Brasil Redes. Método: Estudo exploratório, transversal, de abordagem quali-quantitativa, na forma de casos múltiplos, usando informações disponíveis nos sítios eletrônicos e mídias sociais. Resultados: Informações e iniciativas de tele-educação voltadas à COVID-19 estavam presentes em 15 dos 23 Núcleos existentes. Verificouse produção de amplo leque de materiais diversos, que incluem cursos a distância, webpalestras, webaulas, cartilhas, infográficos e outros materiais informativos. Destaca-se a existência de forte tendência das ações de teleducação com foco nas redes sociais (YouTube, Facebook, Twitter e Instagram), como coadjuvantes na divulgação de produtos e serviços para a população, fortalecendo a democratização e o acesso a informações úteis para usuários, enfermeiros e profissionais dos serviços de saúde. Conclusão: Acesso à informação de forma ágil e transparente, durante o isolamento social é crítico no enfrentamento da pandemia. A web revela-se como importante ferramenta para obter informações sobre a COVID nos Núcleos de Telessaúde. Os resultados mostram papel relevante a ser exercido pelos serviços de telessaúde, considerando a necessidade de qualificação profissional e de acesso à informação de qualidade demandada pela pandemia. Descritores: Telemedicina; Infecções por Coronavirus; Mídias Sociais; Acesso à Informação.

\begin{abstract}
Objective: To identify the health education and information initiatives aimed at coping with the COVID-19 pandemic, offered by state Telehealth Centers associated with the Brazilian Telehealth Network Program. Method: This is an exploratory, cross-sectional study with a qualitative and quantitative approach, in the form of multiple case study, using information available on websites and social media. Results: COVID-19-related Information and tele-education initiatives were present in 15 of the 23 existing Centers. The production of a wide range of different materials was verified, including online courses, web lectures, manuals, infographics, and other informational material. It is worth mentioning the existence of a strong trend in tele-education actions focused on social networks (YouTube, Facebook, Twitter, and Instagram) as assistants in the disclosure of products and services to the population, strengthening democratization and access to useful information for users, nurses and health service professionals. Conclusions: Access to information in a fast and transparent way during social isolation is critical in facing the pandemic. The websites are an important tool to obtain information about COVID-19 on Telehealth Centers. The results show an important role to be played by telehealth services, considering the need for professional qualification and access to quality information required by the pandemic.
\end{abstract}

Key Words: Telemedicine; Coronavirus Infections; Social Media; Access to Information.

\section{RESUMEN}

Objetivo: Identificar las iniciativas de teleducación e información de salud destinadas a hacer frente a la pandemia de SARS-CoV-2 ofrecidas por los Centros estaduales de Telesalud vinculados al "Programa Telessaúde Brasil Redes". Método: Estudio exploratorio, transversal, con enfoque cuali-cuantitativo, en forma de casos múltiples, con base en sus páginas web. Resultados: Las iniciativas de información y teleducación dirigidas al nuevo coronavirus estuvieron presentes en 15 centros. Se verificó la producción de una amplia gama de materiales diferentes, incluidos cursos a distancia, conferencias web, folletos, infografías y otros materiales informativos. Se destaca la existencia de una fuerte tendencia en acciones de teleducación centradas en redes sociales (YouTube, Facebook, Twitter e Instagram), como asistentes en la difusión de servicios a la población, fortaleciendo la democratización y el acceso a informaciones útiles para usuarios, enfermeros y profesionales de salud. Conclusión: El acceso a la información ágil y transparente durante el aislamiento social es crítico para enfrentar la pandemia. La web se revela como una herramienta importante para obtener información sobre SARS-CoV-2 en estos centros. Los resultados muestran un papel relevante de los servicios de telesalud, considerando la necesidad de calificación profesional y el acceso a la información de calidad requerida por la pandemia.

Descriptores: Telemedicina; Infecciones por Coronavirus; Medios de Comunicación Sociales; Acceso a la Información.

'Doutora em Saúde Coletiva. Professora associada do Departamento de Políticas Públicas, Planejamento e Administração em Saúde do Instituto de Medicina Social da Universidade do Estado do Rio de Janeiro - IMS/UERJ. ${ }^{2}$ Doutora em Saúde Pública pela Escola Nacional de Saúde Pública Sergio Arouca. Pesquisadora da Fundação Oswaldo Cruz. ${ }^{3}$ Doutor em Saúde Coletiva pelo Instituto de Medicina Social da UERJ. Docente da Fundação Oswaldo Cruz. ${ }^{4}$ Doutoranda em Saúde Coletiva IMS/UERJ. ${ }^{5}$ Doutoranda em Biociências na UERJ, Coordenadora do Núcleo de Telessaúde do Hospital Federal dos Servidores do Estado. Mestre em Administração de Saúde Instituto de Medicina Social da UERJ. ${ }^{7}$ Doutora em Saúde Coletiva. Professora do Centro Universitário Arthur Sá Earp Neto. ${ }^{8}$ Doutora em Informação e Comunicação. Pesquisadora do grupo de pesquisa Telessaúde e Sistemas de Saúde. ${ }^{9}$ Doutora em Saúde Coletiva pelo IMS/UERJ. 


\section{INTRODUÇÃO}

$\mathrm{Na}$ virada de 2020, o mundo foi surpreendido por uma nova doença, a COVID-19 que, rapidamente, se expandiu para uma pandemia de proporções significativas e com impactos negativos muito relevantes na saúde e na economia. Causada pelo vírus SARS CoV- 2 , seu agente etiológico foi identificado no continente asiático, no final de dezembro de 2019. Em 11 de março de 2020, a doença tinha tal disseminação geográfica que foi caracterizada pela Organização Mundial da Saúde (OMS) como uma pandemia (1).

Os números relacionados à COVID-19 apontam para uma emergência de saúde global. Segundo dados da OMS, até 01 de agosto de 2020, existiam 17.396.943 casos confirmados em todo o mundo, com 675.060 mortes ${ }^{(2)}$. Na mesma data, o Brasil possuía 2.610 .102 casos e 91.263 vítimas fatais ${ }^{(2)}$, o que torna o país um dos epicentros da doença, ainda mais, considerando-se que existe substancial subnotificação de casos e óbitos.

Por se tratar de um vírus novo e uma condição nosológica antes inexistente, o conhecimento sobre a doença tem se construído, paulatinamente, tornando-se um desafio controlar sua disseminação e minimizar seus impactos negativos na saúde da população e dos trabalhadores dos serviços assistenciais e essenciais. Recomendações médicas e comprovações científicas estão em andamento em todo o mundo e muitos esforços têm sido empreendidos, internacionalmente e no Brasil, para respaldar a população, os profissionais de saúde e os gestores para seu adequado enfrentamento. Isso se faz ainda mais relevante, porque, até o presente, não há terapia eficaz ou vacina contra o vírus, com o controle da COVID-19 repousando, fortemente, em medidas amplas de isolamento, quarentena e restrição do contato social. Essas ações são fundamentais para que ocorra uma desaceleração da propagação da epidemia (achatamento da curva de transmissão), protegendo aqueles com maior risco de quadros graves contra a infecção e reduzindo o pico de necessidade por assistência médica ${ }^{(3)}$. Igualmente importante é a utilização de medidas preventivas, como o uso de máscaras, equipamentos de proteção individual e atenção rigorosa a cuidados de higiene pessoal e do ambiente ${ }^{(4)}$.

Em meio à avalanche de relatórios sobre a disseminação do vírus e seus impactos em termos de casos e óbitos, há também o reconhecimento de que a Telessaúde pode desempenhar um papel crítico na resposta global ${ }^{(5)}$. A Telessaúde pode garantir e ampliar o acesso às ações e cuidados em saúde, por meio do atendimento a distância entre prestadores de serviços e usuários, por meio das Tecnologias de Informação e Comunicação (TIC). Ela permite a interação entre os diversos profissionais de saúde, bem como o acesso remoto a recursos de apoio educacional, clínico e diagnóstico ${ }^{(6)}$.

Seu uso na epidemia serviria à triagem, cuidado e tratamento remotos, auxiliando a prevenção, vigilância, detecção e monitoramento e, contribuindo também para mitigar impactos nos cuidados de saúde indiretamente relacionados à COVID-19(7). Sua utilidade, contudo, não se limita às atividades de cunho estritamente assistencial. Comunicação efetiva, de qualidade e confiável é crítica, durante emergências sanitárias como o surto da COVID-19. Planos oficiais de comunicação devem promover facilidade e canais diversos para informar às pessoas sobre a pandemia, evitar rumores, e reduzir iatrogenias e ameaças à saúde pública. Plataformas de mídia social são benéficas para modelar as tendências da doença e para monitorar a evolução dos sintomas dos pacientes ou a reação do público à pandemia ao longo do tempo ${ }^{(8)}$.

No Brasil, país de dimensões continentais e com profundas diferenças regionais, as ações de Telemedicina tiveram início nos anos 90, acompanhando tendência mundial de atendimento médico e geração de laudos à distância, aprimorada pelo recurso bidirecional de vídeo e áudio. Posteriormente, o uso das TIC foi estendido para suportar serviços e atividades de treinamento e de informação em saúde para provedores assistenciais multidisciplinares e pacientes, configurando um campo mais ampliado denominado Telessaúde ${ }^{(7)}$.

Dentre as iniciativas implementadas na agenda pública do país, destacam-se a Rede Universitária de Telemedicina (RUTE) e o Programa Telessaúde Brasil Redes. A RUTE, coordenada pela Rede Nacional de Ensino e Pesquisa (RNP), foi instituída em 2006 com o objetivo principal de aprimorar a infraestrutura de comunicação e colaboração interinstitucional entre hospitais universitários e instituições de saúde ${ }^{(9)}$. A implantação do Programa Telessaúde Brasil Redes se iniciou no ano 2007, com o objetivo de fortalecer e melhorar a qualidade do atendimento da atenção básica no Sistema Único de Saúde (SUS). Foi, inicialmente, implantado como um projeto piloto - Programa Telessaúde 
Brasil - em nove estados: Amazonas, Ceará, Pernambuco, Rio de Janeiro, Minas Gerais, Goiás, São Paulo, Santa Catarina e Rio Grande do Sul ${ }^{(9)}$. Foi redefinido e ampliado por meio da Portaria MS no 2.546, publicada em 27 de outubro de $2011^{(10)}$, passando a ser denominado Programa Telessaúde Brasil Redes. Na atualidade, é constituído por Núcleos Estaduais, presentes em quase todas as unidades da federação, além de núcleos Intermunicipais e Regionais. Esse Programa visa a integrar ensino e serviço, por meio das TIC, para melhoria da qualidade do atendimento, por meio das atividades de educação permanente e da oferta de apoio assistencial, por meio dos Núcleos de Telessaúde (NT) ${ }^{(11)}$.

Os NT são responsáveis pela gerência e coordenação de ações, ofertas e serviços de Telessaúde, como Teleconsultoria (consulta entre trabalhadores, profissionais e gestores da área de saúde, por meio de instrumentos de telecomunicação bidirecional), Telediagnóstico (orientado à relação médico-paciente em função do diagnóstico de condições ou doenças, feito a distância), Segunda Opinião Formativa (teleconsultoria assíncrona sobre dúvidas correntes de profissionais de saúde, que são respondidas e depois validadas por especialistas, seguidas pela difusão na Biblioteca Virtual em Saúde), e Teleducação. Essas diversas modalidades possuem a Atenção Primária à Saúde (APS) como foco de atuação para a qualificação e o fortalecimento da força de trabalho, em especial as equipes multidisciplinares de saúde da família, favorecendo sua interação com os demais níveis de atenção e fortalecendo as Redes de Atenção à Saúde do SUS ${ }^{(10)}$.

A teleducação é modalidade instituída de modo expressivo nas ações de Telessaúde, no país, com o objetivo de qualificar os diferentes trabalhadores da área da saúde e orientar a população. Em 2017, era ofertada em 24 dos 47 núcleos de telessaúde estaduais e intermunicipais implantados. Contribui para potencializar programas de educação permanente, possibilitando o desenvolvimento de profissionais de saúde com postura crítica, comprometidos com a qualidade do cuidado e mais sensíveis a mudanças das práticas de atenção e da organização dos processos de trabalho ${ }^{(10,12)}$. Para tal, a teleducação utiliza-se de um conjunto amplo de ações educativas (conferências, webaulas, palestras, cursos, fóruns de discussão, bate-papos, dentre outros) mediadas pelas TIC, que podem fortalecer a atenção à saúde no SUS ${ }^{(10,12)}$.
Esse estudo faz parte de um projeto de pesquisa intitulado "Modelo de avaliação de serviços de Telessaúde (MAST): adaptação e validação para o contexto brasileiro", cuja proposta é elaborar um modelo de avaliação que contribua para a ampliação de estratégias de acompanhamento, monitoramento e avaliação das ações de Telessaúde no SUS. Uma das etapas do projeto envolveu o mapeamento das aplicações de Telessaúde oferecidas pelos Núcleos de Telessaúde, no Brasil e, durante sua realização, o surgimento da pandemia da COVID-19 ofereceu uma oportunidade de redirecionar os esforços dos pesquisadores, no sentido de observar as iniciativas de informação e educação em saúde, mediadas pelas ferramentas de internet e pelas TIC para contribuir no enfrentamento da nova doença.

Por se tratar de um vírus e uma doença novos no cenário mundial, a necessidade de produção e divulgação do conhecimento sobre a COVID-19 para os trabalhadores do SUS e para a população revela-se como absolutamente imprescindível. A teleducação configura-se como uma ferramenta para a construção e aperfeiçoamento desse conhecimento, podendo promover acesso à informação de qualidade, respaldada em evidências e produzida por órgãos oficiais de referência nacional e mundial. Diante da atual conjuntura, torna-se relevante dar visibilidade e destacar as ações e práticas de teleducação, considerando-as como um componente estratégico para o combate à pandemia ainda em curso.

Assim, neste artigo, objetivou-se identificar e sistematizar as iniciativas de educação e de divulgação de informações em saúde direcionadas ao enfrentamento da pandemia pela COVID-19 produzidas pelos Núcleos de Telessaúde vinculados ao Programa Telessaúde Brasil Redes.

\section{MÉTODOS}

Trata-se de uma pesquisa exploratória, transversal e de abordagem quali-quantitativa, que se utilizou do método de estudo de casos múltiplos. Estudos desse tipo permitem a utilização de várias abordagens metodológicas de forma complementar e investigar, empiricamente, um fenômeno contemporâneo dentro de seu contexto de vida real ${ }^{(13)}$. Na forma de casos múltiplos, adiciona a vantagem de uma exploração mais abrangente e com possibilidade de comparação, aspecto importante, considerando-se a diversidade de serviços de 
Telessaúde presentes no país.

O estudo de casos múltiplos foi orientado pela seguinte questão: Que tipo de iniciativas de educação e informação em saúde estão sendo utilizadas pelos Núcleos de Telessaúde para instruir a população e qualificar os profissionais que atuam no contexto da pandemia? Seguindo recomendações teóricas e metodológicas ${ }^{(13)}$, o estudo foi desenvolvido, observando-se quatro etapas básicas: (1) planejamento do estudo; (2) coleta de dados; (3) descrição individual dos casos, e (4) análise e sistematização dos dados.

Na primeira etapa, os Núcleos Estaduais de Telessaúde que integram o Programa Telessaúde Brasil Redes foram definidos como os casos a serem analisados. A opção por se concentrar nos núcleos estaduais partiu do pressuposto que estes, ao representarem a maior instância em termos das unidades da federação, possuiriam maiores condições, em termos de estrutura e recursos humanos, para se organizar e responderem mais prontamente às necessidades e à urgência ditadas pela epidemia em expansão. Ademais, todos esses Núcleos possuem a teleducação como uma de suas atividades centrais, em decorrência desta exigência normatizada pela Portaria MS no 2.546 de 2011. Normativas também definem que as atividades de teleducação devem ser registradas em plataformas on line, onde é possível cadastrar usuários e estabelecimentos que utilizam esses serviços ${ }^{(14)}$.

Todos os Núcleos Estaduais pertencentes ao Programa Telessaúde Brasil Redes foram identificados partindo do disposto na página eletrônica do Ministério da Saúde, que traz uma listagem com os respectivos endereços eletrônicos ${ }^{(11)}$. O levantamento das informações e das iniciativas divulgadas como ações de teleducação foi realizado, acessando os sítios dos Núcleos na web e suas redes sociais oficiais (Facebook: https://ptbr.facebook.com/pg/facebook/about/; YouTube: https://www.youtube.com/intl/pt-PT/about/; Instagram: https://about.instagram.com/ptbr/about-us; Twitter: https://about.twitter.com/pt.html).

A produção de conteúdo de informação e educação em saúde sobre a COVID-19 foi considerada como a unidade de análise do estudo, tomando por base as atividades de teleducação contidas no momento da coleta de dados e sendo aplicada a técnica da observação direta ${ }^{(13)}$ das páginas eletrônicas e mídias sociais dos NT. Essa técnica de observação é útil quando "trata-se de observação de acontecimentos em tempo real"(13:108), sendo considerada oportuna para o estudo realizado.

A coleta de dados foi realizada de forma intensiva, nos dias 18 e 19 de maio de 2020. Com o esforço de coleta em curto intervalo, objetivouse trazer um retrato de ações que, pelas suas natureza e características, são adicionadas de forma bastante dinâmica e quase contínua no tempo.

Um formulário de coleta foi construído na forma de planilha no software Exce ${ }^{\circledR}$, contendo as variáveis de interesse: nome do NT; link da página do NT na internet; link da página do hotsite; links das páginas das redes sociais; ações educativas; podcasts; infográficos; cartilhas; manuais, protocolos e notas técnicas; boletins epidemiológicos; conteúdos áudiovisuais disponíveis no YouTube e outras mídias, com seus respectivos temas; webconferências; materiais educativos para a população; aplicativos móveis e jogos. A provisão desse instrumento padronizado ${ }^{13)}$ garantiu que as informações fossem coletadas na web de forma sistemática, rápida e por seis diferentes pesquisadores.

A descrição individual dos casos realizada na terceira etapa contemplou a exportação das informações coletadas por cada pesquisador, de forma individual e independente, para a planilha de dados. Ao final da coleta, os pesquisadores fizeram reuniões via sistema de videoconferência da Rede Nacional de Ensino e Pesquisa (RNP) e verificaram eventuais lacunas informacionais e incorreções, que foram objeto de reverificação por um pesquisador diferente do que havia realizado a coleta. Após essa revisão, todas as informações foram consolidadas em uma única planilha, formando um banco de dados com as evidências encontradas.

$\mathrm{Na}$ quarta e última etapa do estudo, foi realizada a análise por Construção da Explanação, uma das possibilidades "analíticas recomendadas para estudos de casos múltiplos"(13:131). O objetivo dessa abordagem é possibilitar a elaboração de uma explanação ou "explicação" de um fenômeno mediante a análise descritiva das variáveis observadas, considerando estabelecer semelhanças e particularidades relacionadas ao mesmo ${ }^{(13)}$. A explanação foi elaborada de forma narrativa, e complementou-se a discussão dos conteúdos com base nos referenciais presentes na literatura sobre o tema. Por fim, os resultados foram apresentados com o auxílio de quadros e 
gráficos para sistematizar a apresentação das informações coletadas, a fim de dar visibilidade às iniciativas dos NT sobre o tema da COVID-19, tendo em vista sua potencial utilidade pelos profissionais de saúde, principalmente enfermeiros, gestores dos serviços do SUS e população.

As quatro etapas executadas na consecução do trabalho estão sumarizadas, graficamente, na Figura 1, abaixo.

Figura 1 - Etapas de coleta e sistematização das informações e atividades educacionais relativas à COVID-19 presentes nos sítios dos Núcleos Estaduais vinculados ao Programa Telessaúde Brasil Redes.
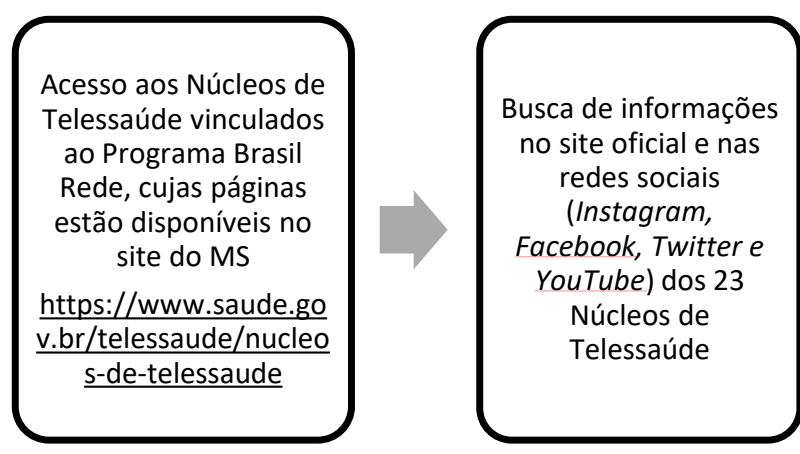

Fonte: Dados da pesquisa, 2020.

A investigação dispensou a apreciação prévia por parte de Comitê de Ética, uma vez que não envolveu pesquisa direta com seres humanos e utilizou apenas fontes públicas de dados secundários e de acesso on-line.

\section{RESULTADOS E DISCUSSÃO}

Os Núcleos Estaduais de Telessaúde do Programa Telessaúde Brasil Redes estão presentes em 23 estados: Acre, Alagoas, Amazonas, Bahia, Ceará, Espírito Santo, Goiás, Maranhão, Minas Gerais, Mato Grosso, Mato Grosso do Sul, Pará, Paraná, Paraíba, Pernambuco, Rio de Janeiro, Rio Grande do Norte, Rio Grande do Sul, Roraima, Santa Catarina, São Paulo, Sergipe e Tocantins.

Todos os NT estaduais possuem páginas oficiais. Em princípio, essa existência favoreceria a transparência e publicização das iniciativas, promovendo o acesso à informação e às produções de educação em saúde. Contudo, não foi possível obter acesso às páginas dos NT do Paraná, Paraíba e de Tocantins. As páginas dos dois primeiros encontravam-se, sistematicamente, fora do ar em repetidas tentativas, mesmo após o término do período de coleta de dados; a página de Tocantins exige cadastro e senha para ter acesso às informações, o que depõe, desfavoravelmente, ao seu amplo acesso.

Nem todos os NT com páginas acessíveis possuíam, contudo, informações e iniciativas de teleducação voltadas para o tema da COVID-19, no momento da pesquisa, como foi o caso de cinco Núcleos: Alagoas, Pará, Roraima, Santa Catarina e Ceará. Esses dois últimos estavam com suas atividades suspensas, no momento da coleta de dados da pesquisa. Dessa forma, a coleta de dados foi feita em 15 NT.

Desses, sete (47\%) correspondem aos primeiros nove Núcleos, inicialmente, implementados pelo Programa Telessaúde Brasil Redes, onde pela longevidade e consolidação era esperada maior facilidade de desencadeamento das ações investigadas. Nos Núcleos da Bahia, Acre, Espírito Santo, Mato Grosso, Mato Grosso do Sul, Sergipe, Rio Grande do Norte e Maranhão também foram identificadas a presença de iniciativas de informação e educação em saúde sobre a COVID-19 (Quadro 1). 
Quadro 1 - Síntese das iniciativas de informação e educação em saúde sobre a COVID-19, produzidas e divulgadas nos sítios dos Núcleos Estaduais de Telessaúde do Programa Telessaúde Brasil Redes, nos dias 18 e 19 de maio de 2020.

\begin{tabular}{|c|c|c|}
\hline $\begin{array}{l}\text { Núcleo de } \\
\text { Telessaúde }\end{array}$ & Iniciativas de teleducação relacionada ao COVID-19 & Link da Página* \\
\hline NT Acre & Teleorientação através do "Disque Coronavírus"; webaulas & http://www.telessaude.ac.gov.br/ \\
\hline NT Amazonas & $\begin{array}{l}\text { Página dedicada ao Coronavírus; informativos; vídeos do MS; } \\
\text { protocolo para APS; cursos de atualização na modalidade EaD } \\
\text { para profissionais }\end{array}$ & https://telessaudeam.org.br/COVID-19/ \\
\hline NT Bahia & $\begin{array}{l}\text { Página dedicada ao Coronavírus; boletins epidemiológicos; } \\
\text { protocolos; notas técnicas e plano de contingência da Bahia; } \\
\text { espaço de dúvidas frequentes; perguntas frequentes e } \\
\text { orientações sobre Fake News; informativos; web palestras; } \\
\text { webaulas; divulgação de cursos de capacitação; podcast; cartilhas } \\
\text { e materiais educativos para APS }\end{array}$ & $\begin{array}{l}\text { http://www.saude.ba.gov.br/temasdesa } \\
\text { ude/coronavirus/ }\end{array}$ \\
\hline $\begin{array}{l}\text { NT Espírito } \\
\text { Santo }\end{array}$ & Web palestras & https://telessaude.ifes. \\
\hline NT Goiás & $\begin{array}{l}\text { Página dedicada ao Coronavírus; informativos; perguntas } \\
\text { Frequentes; webaulas }\end{array}$ & https://covid19.medicina.ufg.br/ \\
\hline NT Maranhão & Web palestras; seminários e chatbot & http://telessaude.huufma.br/portal/ \\
\hline NT Minas Gerais & $\begin{array}{l}\text { Página dedicada ao Coronavírus (hotsite); informações para } \\
\text { profissionais e para o cidadão; vídeos; infográficos; perguntas e } \\
\text { respostas; protocolos para APS e serviços de Urgência; Aplicativos } \\
\text { móveis; Jogos; artigos; boletim epidemiológico; informativos; } \\
\text { biblioteca de textos; links úteis }\end{array}$ & https://www.telessaudemg.net/ \\
\hline NT Mato Grosso & Webaulas & http://www.telessaude.mt.gov.br/ \\
\hline $\begin{array}{l}\text { NT Mato Grosso } \\
\text { do Sul }\end{array}$ & $\begin{array}{l}\text { Página dedicada ao Coronavírus no sítios; informativos; webaulas; } \\
\text { web palestras; plano de contingência; nota técnicas; protocolos } \\
\text { para APS; manuais e documentos do MS }\end{array}$ & $\begin{array}{l}\text { http://telessaude.saude.ms.gov.br/port } \\
\text { al/2020/03/covid-19/ }\end{array}$ \\
\hline NT Pernambuco & $\begin{array}{l}\text { Página dedicada ao Coronavírus; informações para profissionais } \\
\text { de saúde e para o cidadão; aplicativos móveis; boletim } \\
\text { epidemiológico; notas técnicas e web palestras }\end{array}$ & http://telessaude.pe.gov.br/corona/info \\
\hline $\begin{array}{l}\text { NT Rio de } \\
\text { Janeiro }\end{array}$ & $\begin{array}{l}\text { Materiais educativos; protocolos e manuais para profissionais de } \\
\text { saúde; cursos de capacitação na modalidade EaD; podcasts; } \\
\text { chatbot; perguntas e respostas; materiais para a população; } \\
\text { Webinars }\end{array}$ & $\begin{array}{l}\text { http://www.telessaude.ueri.br/teleeduc } \\
\text { acao/course/view.php?id=127 }\end{array}$ \\
\hline $\begin{array}{l}\text { NT Rio Grande } \\
\text { do Norte }\end{array}$ & Cursos para capacitação; webconferências & http://www.telessaude.ufrn.br/ \\
\hline $\begin{array}{l}\text { NT Rio Grande } \\
\text { do Sul }\end{array}$ & $\begin{array}{l}\text { Página dedicada ao Coronavírus; perguntas frequentes; manuais; } \\
\text { protocolos para a APS; Painel Coronavírus e materiais educativos }\end{array}$ & https://www.ufrgs.br/telessauders/ \\
\hline NT Sergipe & $\begin{array}{l}\text { Web palestras; disponibiliza aplicativo móvel para acesso a } \\
\text { atendimento virtual e orientação de médicos }\end{array}$ & https://telessaude.se.gov.br/ \\
\hline $\begin{array}{l}\text { NT de São } \\
\text { Paulo* }\end{array}$ & $\begin{array}{l}\text { Perguntas frequentes; SOF; link direto para acesso a página da } \\
\text { prefeitura de São Paulo onde é possível ter acesso a documentos } \\
\text { técnicos; boletins epidemiológicos; notas técnicas; legislações; } \\
\text { recomendações; manuais do MS e ANVISA; orientações sobre } \\
\text { Fake News e divulgação de cursos de capacitação }\end{array}$ & $\begin{array}{l}\underline{\text { https://telessaude.prefeitura.sp.gov.br/ }} \\
\underline{\text { https://www.prefeitura.sp.gov.br/cidad }} \\
\text { e/secretarias/saude/vigilancia em saud } \\
\underline{\text { e/doencas e agravos/index.php?p=291 }} \\
\underline{766}\end{array}$ \\
\hline
\end{tabular}

Fonte: Dados da pesquisa, 2020 a partir de informações coletadas nos sítios eletrônicos dos Núcleos Estaduais de Telessaúde em 18 e 19 de maio de 2020.

Nota*: O Núcleo de São Paulo é denominado Telessaúde Redes do Município de São Paulo.

Legenda: APS- Atenção Primária à Saúde, SOF- Segunda Opinião Formativa, MS- Ministério da Saúde, EaD- Ensino à Distância, NTNúcleo de Telessaúde, ANVISA- Agência Nacional de Vigilância Sanitária.

Durante o período da pandemia de COVID19, a Telessaúde teve seu uso ampliado no Brasil $^{(7)}$. Nesse período, o país se mobilizou para seu enfrentamento com a implementação de leis e normativas que ressaltam a importância da Telessaúde, nesse cenário, principalmente no âmbito da teleassistência(15-16). A teleconsulta sem visita presencial prévia, até então só permitida pelo Conselho Federal de Medicina em casos excepcionais, destaca-se como uma inovação não presente no contexto nacional. Nesse sentido, a Portaria MS no 467, de 23 de março de 2020, autoriza sua prática - em caráter de excepcionalidade - em ações que contemplem o atendimento pré-clínico, de suporte assistencial, de consulta, monitoramento e diagnóstico, no 
âmbito do SUS, saúde suplementar e privada (Art. 2oํ) $)^{(15)}$.

A Telessaúde tem favorecido as atividades
de teleducação, permitindo mesclar o conhecimento científico com o conhecimento empírico, proporcionando uma visão crítica e maior participação, e melhorando o acesso a estratégias e materiais educacionais. As iniciativas de informação e de educação em saúde mediadas pelas TIC produzidas pelos NT merecem relevância, no contexto da pandemia. A existência de materiais diversificados para enfermeiros e outros profissionais de saúde, recursos audiovisuais, aplicativos e hotsites ${ }^{(17)}$, dentre outras iniciativas, vêm sendo utilizados para a estruturação e divulgação de informações em saúde sobre a COVID-19.

Em sete NT (Minas Gerais, Pernambuco, Goiás, Amazonas, Bahia, Rio Grande do Sul e Mato Grosso do Sul), rearranjos visuais parecem ter ocorrido nos seus sítios da web, com a página de entrada do NT apresentando áreas específicas dedicadas ao coronavírus, onde é dado destaque aos conteúdos informativos relacionados ao tema da COVID-19. A presença de cores vibrantes dos hotsites (sítios criados temporariamente para divulgarem informações sobre serviços e campanhas), e de imagens vinculadas ao coronavírus funcionam, ademais, como estratégias para chamar atenção daqueles que acessam os sítios em busca das informações produzidas sobre o tema ${ }^{(17)}$.

A produção de material educativo audiovisual mostrou-se bastante intensiva e difusa pelos NT, incluindo webpalestras, webinars, webaulas, webconferências, cursos, seminários e materiais infográficos, que podem ser acessados pelos interessados. As temáticas abordadas, nesse conjunto de iniciativas educacionais, são extremamente diversificadas, incluindo, desde informações sobre sinais e sintomas da COVID-19; interpretação dos testes para o diagnóstico da doença; medidas preventivas como higienização de mãos, uso de máscaras e equipamentos de proteção individual, até cuidados específicos relacionados à saúde mental e ao pré-natal; violência contra a pessoa idosa; saúde da mulher, da população negra e de crianças e adolescentes; atenção à população indígena e aos pacientes portadores de doenças raras, todas no contexto da pandemia em curso.

Grande parte dessa produção é direcionada para os profissionais de saúde, coerente com o compromisso do Programa de Telessaúde Brasil
Redes, como estratégia de educação continuada. Reitera-se que tais ações devem ser pensadas e ofertadas na perspectiva de um projeto maior, que considere as demandas e necessidades das equipes, incentivando, permanentemente, a identificação de problemas no contexto de trabalho, a ampliação do conhecimento aplicado ao espaço local, o trabalho interdisciplinar e o protagonismo das equipes na tomada de decisão (12). Ao mesmo tempo, foram identificadas iniciativas que buscam informar a população para sua autonomia e autocuidado, como a produção de cartilhas, infográficos e outros materiais, como, por exemplo, recursos específicos direcionados a crianças e para gestantes presentes na página do NT do Rio de Janeiro. Reforça-se, assim, o papel social, deste trabalho, mediado pelas TIC, pois essas ferramentas já estabelecidas para a educação profissional passam a ser usadas mais amplamente para a educação em saúde da sociedade ${ }^{(18)}$.

Outros documentos como guia de práticas e condutas, informativos, manuais clínicos, protocolos e boletins epidemiológicos também são fontes de informação importantes para instrumentalizar e auxiliar os profissionais no atendimento, manejo clínico, organização do fluxo assistencial, notificação, dentre outras ações necessárias para o enfrentamento da pandemia. Nesse sentido, observa-se que a disponibilização desses documentos potencialmente ajuda a divulgar e orientar diretrizes para os diferentes níveis de atenção dentro do sistema de saúde, podendo contribuir para a melhoria da assistência. Os materiais produzidos são de acesso aberto, podem ser resgatados por download e compartilhados, gratuitamente, nos sítios dos NT.

Diversos NT, como, por exemplo, o do Rio Grande do Sul, possuem seções específicas de perguntas frequentes, onde buscam abordar questões práticas que os profissionais precisam manusear, nesses tempos pandêmicos como, por exemplo, aplicabilidade dos testes diagnósticos para COVID-19, investigação de COVID-19 após o óbito em casos suspeitos, preenchimento da declaração de óbito em casos suspeitos ou confirmados de coronavírus, orientações sobre o atendimento odontológico na APS, no contexto da epidemia, dentre outros, com referências científicas que permitem 0 aprofundamento posterior do profissional interessado.

Os painéis eletrônicos de dados, disponibilizados na internet por alguns dos NT estaduais, contribuem na disseminação de 
informação epidemiológica atualizada sobre os números de casos suspeitos, confirmados, recuperados e de óbitos relacionados à doença, frequentemente apresentando suas distribuições espaciais pelos municípios ou regiões sanitárias dos estados. A divulgação desse monitoramento constante por meio das TIC também é realidade em outras instituições isoladas, trazendo dados diários e, por vezes, em tempo real, sobre casos confirmados e suspeitos de COVID-19, mortes, internações em UTI e pacientes em ventilação mecânica ${ }^{(7)}$.

0 recurso de áudio, por meio de podcast é um artifício utilizado pelos NT do Amazonas, Rio de Janeiro e da Bahia para transmitir informações e facilitar o alcance, pois o material pode ser acessado via internet pelo aparelho celular e estar acessivel a deficientes visuais. Os podcasts proporcionam maior integração junto ao público e temas abordados por essa modalidade incluíram: "Dúvidas mais comuns sobre a pandemia de Covid-19", "Saúde mental em tempos de pandemia", "Como combater as fake news através da busca por informações confiáveis", "Efeitos pós-pandemia em diversos grupos sociais" e "Testes e vacinas para Covid-19".

Dentre as iniciativas digitais encontradas, neste levantamento, merece menção um jogo sobre a COVID-19 produzido pelo NT de Minas Gerais, apresentando conteúdos educativos sobre prevenção e contaminação que permitem à população adquirir conhecimento respaldado em base científica de forma confiável e, ao mesmo tempo, divertida e estimulante. $O$ objetivo do jogo é promover mudanças de atitude que reduzam a transmissão do novo coronavírus. Essa contribuição para a sociedade é uma ação de extensão da Faculdade de Medicina da UFMG, que disponibiliza o acesso ao jogo em diversos formatos digitais: computador, tablet, smartphones e, até mesmo, em aparelhos de celular mais simples para facilitar o acesso. A utilização de elementos de design de jogos em contextos não relacionados a jogos é uma estratégia apontada em estudos como meio de aumentar a inicialização e retenção dos comportamentos de saúde desejados, estimulando o envolvimento dos participantes e facilitando seu aprendizado sobre saúde ${ }^{(19)}$.

Outra iniciativa apresentada nos múltiplos recursos disponibilizados pelos NT refere-se a conteúdo específico direcionado às crianças, obrigadas a ficar reclusas e afastadas das atividades escolares, durante o isolamento social.
O NT do Rio de Janeiro, por exemplo, apresenta material elaborado com linguagem simples e direta, atendendo às especificidades do público infantil e contendo atividades lúdicas simples, como colorir e caça-palavras, o que contribui para auxiliar pais e educadores no tratamento do tema. Estratégias similares têm sido desenvolvidas por outros atores institucionais, como a iniciativa da Fundação Oswaldo Cruz (Fiocruz), que já possui dez "livretos de Quarentena" disponibilizados em seu sítio eletrônico, onde não apenas são trazidas informações sobre a doença e sua prevenção, mas outros apoios familiares como sugestões de atividades a serem desenvolvidas com os pequenos, tais como: receitas, brincadeiras, quiz e outros ${ }^{(20)}$.

O telefone ganhou destaque em tempos de isolamento social, pois a interatividade de modo contínuo e instantâneo, através de chat, chatbot (programas que simulam uma conversa, como as estabelecidas entre seres humanos, podendo ser utilizados com os mais diversos propósitos, inclusive para a área de saúde ${ }^{(17,21)}$, e do aplicativo de mensagens Whatsapp [https://web.whatsapp.com/] como recursos direcionados a monitorar pacientes, orientar profissionais de saúde e a população sobre os cuidados em saúde. $O$ uso dessas ferramentas foi observado em nove dos 15 NT (Acre, Bahia, Goiás, Maranhão, Minas Gerais, Pernambuco, Rio de Janeiro, Sergipe e São Paulo).

Com o cenário imposto pela pandemia, o uso do WhatsApp tem se potencializado como uma ferramenta de comunicação. É frequente seu uso pelos enfermeiros e outros profissionais de saúde para a difusão de informações de saúde, troca de informações e tomada de decisão clínica entre os profissionais, apoio social a pacientes, durante tratamento e disseminação de orientações de saúde ${ }^{(22)}$. Para evitar vazamentos e exposição, a plataforma de comunicação entre profissionais de saúde e pacientes necessita de uma infraestrutura tecnológica adequada com segurança digital, respeitando a Lei Geral de Proteção de Dados Pessoais, a fim de garantir a privacidade dos dados do paciente e a confidencialidade das informações trocadas na teleconsulta. Contudo, cabe destacar que o registro realizado nesse aplicativo de mensagens e chamadas não substitui o registro dessa informação no prontuário do paciente, seja ele eletrônico ou impresso.

No âmbito internacional, o telefone já era utilizado em telemedicina para a vigilância de 
riscos à saúde. No atual momento, sua aplicabilidade se intensificou, em razão da gravidade da doença e necessidade de distanciamento social imposta pela COVID-19. Há que se levar em conta, entretanto, as normativas éticas e legais para as consultas e informações concedidas por telefone e por vídeo. Nesse sentido, é preciso considerar a organização do acompanhamento clínico e das próximas etapas do cuidado (22).

Sítios de mídia social têm emergido como fontes populares de informações sobre saúde, especialmente para adolescentes e jovens adultos, pelas vantagens como o baixo custo, transmissão rápida, através de uma ampla comunidade e interação do usuário. Nas páginas oficiais dos 15 NT são encontrados ícones que redirecionam para redes sociais como Instagram, Twitter, YouTube e Facebook. O ícone direcionado ao Facebook estava presente em todos eles.

Essas redes sociais são coadjuvantes no processo de divulgação das atividades produzidas e, muitas vezes, há duplicidade de informações nesses ambientes, que tendem a aumentar o alcance da propagação dos conteúdos tratados. Elas são utilizadas pelos NT tanto para divulgar as iniciativas e disseminar os conteúdos informacionais produzidos pelos Núcleos quanto também para facilitar o contato com os interessados nas atividades oferecidas. $O$ uso desses recursos, por meio de diferentes plataformas, pode contribuir para o fortalecimento e ampliação do compartilhamento das informações de saúde. Porém, certamente, a efetividade desse uso nos cenários descritos envolve, sobretudo, a apropriação desses recursos pela população como parte do seu cotidiano comunicacional de vida. Se todos já usam os aplicativos e redes sociais para falar com seus familiares e amigos, é natural que se estenda seu uso para a comunicação profissional ${ }^{(18)}$.

Assim, dentro do contexto da informação e comunicação baseada em evidência, faz-se necessário problematizar a qualidade da informação em saúde veiculada pelas redes sociais. Um dos fenômenos que tem ganho ascendência na atualidade, e não apenas relacionado ao coronavírus e à pandemia, são as Fake News. Estas são definidas como notícias falsas, sem evidência científica e veiculadas como verdade na imprensa escrita e nas mídias sociais $^{(23)}$.

Com o intuito de combater essa prática, foram criados conteúdos nos NT com perguntas e respostas, bem como espaços de esclarecimento de dúvidas, para combater as notícias falsas sobre a COVID-19. Essas iniciativas fortalecem que as informações em saúde sejam válidas e confiáveis, bem como difundidas de forma segura. Ainda nessa linha de atuação, observam-se esforços governamentais para combater notícias falsas. 0 Ministério da Saúde, por exemplo, abriu um canal de comunicação com a população através do WhatsApp - (61) 99289-4640 e, adicionalmente, dispõe de profissionais de saúde nas áreas técnicas para confirmar se a notícia é verdadeira ou falsa. O mesmo se verifica em diversas secretarias estaduais de saúde, inclusive algumas aos quais os NT estão vinculados. Nesse sentido, a disponibilização adicional dos espaços acima mencionados pelos NT, bem como a divulgação de materiais como diretrizes e informações técnicas em suas páginas e mídias sociais, vem se somar às iniciativas governamentais, fortalecendo o combate à veiculação de informações falsas, que só confundem profissionais e a população em geral e podem ter efeitos bastante danosos para a consecução do adequado combate à epidemia e seus efeitos.

Percebeu-se que os NT divulgam conteúdos de educação em saúde, em diversos formatos e que o principal meio de divulgação é feito através do YouTube. Com mais de 2 bilhões de usuários, o YouTube é um canal de mídia, ao qual milhões de pessoas recorrem a fim de buscar informações. Nesse sentido, é importante considerar que o conteúdo disponibilizado pelos NT, neste canal, permite alcançar muitos indivíduos, com o compartilhamento e comentários, sendo recursos permitidos por essa rede social, o que aumenta a visibilidade e o alcance da informação. Em uma amostra de 100 vídeos mais vistos no YouTube sobre o coronavírus em janeiro de 2020, pesquisadores identificaram que eles foram assistidos mais de 125 milhões de vezes e até março já contabilizavam mais de 165 milhões de visualizações ${ }^{(24)}$.

Dos quinze Núcleos, catorze disponibilizavam vídeos das webpalestras, webconferências e webaulas sobre temas relacionados ao coronavírus, que podem ser acessados tanto pelos profissionais quanto pela população em geral. A semelhança e recorrência dos conteúdos produzidos pelos NT permitiram o agrupamento das informações na Figura 2. 
Figura 2 - Temas presentes nos conteúdos educativos sobre a COVID-19 divulgados pelos Núcleos de Telessaúde do Programa Telessaúde Brasil Redes em seus canais no YouTube, nos dias 18 e 19 de maio de 2020.

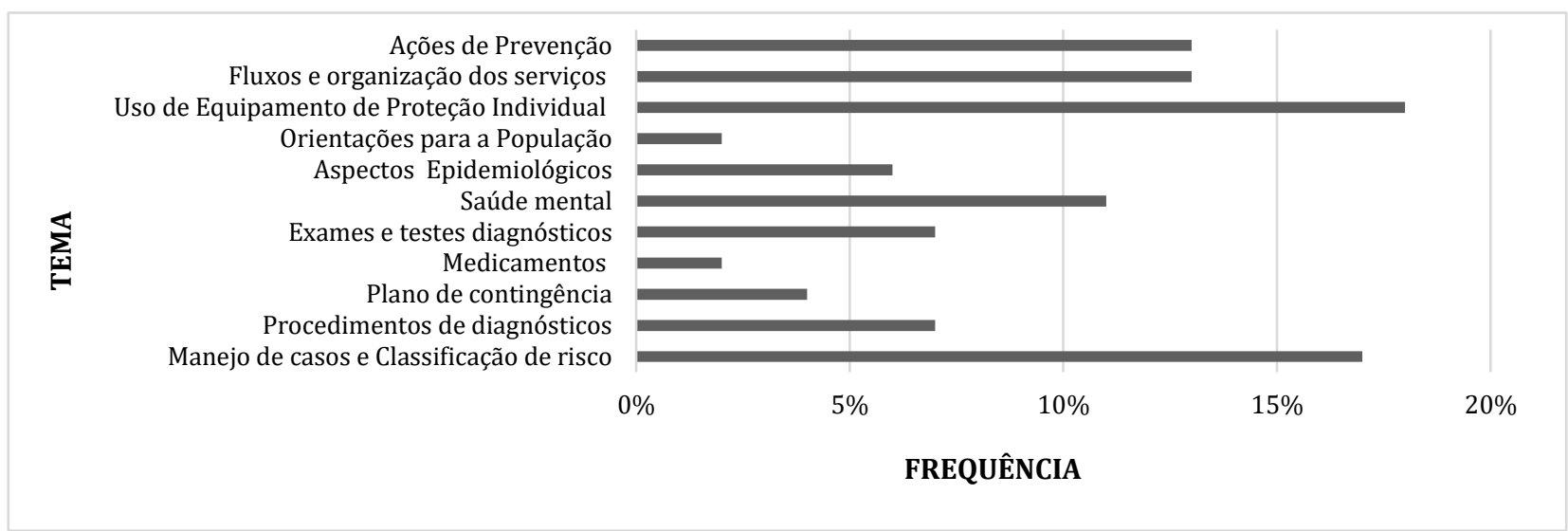

Fonte: Dados da pesquisa, 2020.

Em relação às temáticas, observa-se que grande parte dos conteúdos dos vídeos das webaulas e webpalestras direcionadas para profissionais foca em aspectos do uso na paramentação e desparamentação dos Equipamentos de Proteção individual (EPI) e no manejo clínico e classificação de risco dos pacientes. São conteúdos extremamente oportunos e relevantes, se considerarmos o grande número de enfermeiros e profissionais de saúde infectados, desde o início da pandemia no país, muitos dos quais perderam suas vidas no combate à COVID-19, e a escassez de recursos estruturais, em termos de testes diagnósticos, leitos e recursos humanos, presentes, de forma constante, em todas as regiões do Brasil.

Temáticas, abordando as ações de prevenção da doença foram encontradas em 13
NT. Sabe-se que comportamentos de proteção e higiene pessoais adequados, são a melhor maneira de prevenir a transmissão de doenças e a COVID-19 ${ }^{(24)}$. Logo, os conteúdos dos vídeos sobre cuidados no ambiente doméstico, higienização das mãos e outros hábitos de higiene são de grande relevância para mitigação da disseminação dessa infecção viral. Tais recomendações apresentadas em vídeos podem ser uma alternativa útil para oferecer as informações ao público com baixo nível de alfabetização ${ }^{(24)}$.

A variedade de informações sobre a COVID19 produzidas por alguns NT contemplava um amplo espectro de assuntos, ressaltando-se que os temas tratados tinham sempre como escopo cuidados específicos relacionados à pandemia, conforme pode ser visto no Quadro 2.

Quadro 2 - Conteúdos temáticos trazidos nos vídeos disponibilizados pelos Núcleos de Telessaúde do Programa Telessaúde Brasil Redes, em seus canais no YouTube, nos dias 18 e 19 de maio de 2020.

\begin{tabular}{|l|l|}
\hline Núcleos de Telessaúde* & \multicolumn{1}{|c|}{ Conteúdos temáticos dos vídeos sobre os cuidados de saúde em tempos de pandemia } \\
\hline Acre & $\begin{array}{l}\text { Atenção ao pré-natal e parto e pós-parto; cuidados do recém-nascido; nutrição; ortotanásia e } \\
\text { distanásia }\end{array}$ \\
\hline Amazonas & Gestação; câncer e seu manuseio na epidemia; amamentação \\
\hline Bahia & Nutrição e sistema imunológico; cuidados à saúde do idoso; atendimento odontológico \\
\hline Espírito Santo & Avaliação radiológica dos pacientes suspeitos ou com a doença; conjuntivite viral \\
\hline Goiás & Nutrição e cuidados nas compras de alimentos \\
\hline Minas Gerais & $\begin{array}{l}\text { Síndromes respiratórias associadas ao COVID-19; síndrome gripal; síndrome gripal X síndrome } \\
\text { respiratória aguda grave }\end{array}$ \\
\hline Maranhão & Saúde do idoso; agenda positiva para crianças, síndrome respiratória aguda grave \\
\hline Mato Grosso do Sul & $\begin{array}{l}\text { Mulheres vítimas de violência; alimentação e nutrição adequada; rede psicossocial; atenção à saúde } \\
\text { materno-infantil }\end{array}$ \\
\hline
\end{tabular}




\begin{tabular}{|l|l|}
\hline Núcleos de Telessaúde* & \multicolumn{1}{|c|}{ Conteúdos temáticos dos vídeos sobre os cuidados de saúde em tempos de pandemia } \\
\hline Mato Grosso & $\begin{array}{l}\text { Assistência à gestação e ao parto; tratamento odontológico; fisioterapia; autocuidado; aleitamento } \\
\text { materno; práticas integrativas e complementares; orientação para Agente Comunitário de Saúde nas } \\
\text { visitas domiciliares; síndromes respiratórias; recomendações para Atenção Primária à Saúde; } \\
\text { particularidades no cuidado à saúde da criança, cuidados paliativos }\end{array}$ \\
\hline Pernambuco & Cuidados pós-morte; cuidados no o sistema prisional \\
\hline Rio de Janeiro & Cuidados pós-morte \\
\hline Rio Grande do Norte & Saúde do Idoso; cuidados na pandemia; ciência do enfrentamento \\
\hline São Paulo & $\begin{array}{l}\text { Protocolos para Serviço de Atendimento Móvel de Urgência; atendimento ao paciente em Parada } \\
\text { Cardiorrespiratória; protocolos para a COVID-19 }\end{array}$ \\
\hline Sergipe & $\begin{array}{l}\text { Assistência Humanizada durante a COVID-19: Pré-natal, Parto, Nascimento e Puerpério; Sistema } \\
\text { Prisional: vigilância, manejo e fluxo de atendimento; aleitamento materno; isolamento social; } \\
\text { controle de qualidade de amostras para teste de COVID-19 }\end{array}$ \\
\hline
\end{tabular}

Fonte: Dados da pesquisa, 2020.

Nota: * No Rio Grande do Sul, o canal do YouTube não apresentava vídeos sobre a COVID-19 no momento da pesquisa. No Instagram, o Núcleo trazia um comunicado sobre a preparação de vídeos educativos que seriam disponibilizados nos sites e redes oficiais em breve para toda população.

Todas as informações, conteúdos e orientações sobre a COVID-19 são de utilidade pública. Assim, os ambientes virtuais, mídias e redes sociais como Facebook, WhatsApp, Instagram e YouTube favorecem a disseminação da informação. Essas redes são usadas por uma considerável parcela da população e projetam-se como estratégicas para otimizar e favorecer o acesso das pessoas a tais conteúdos ${ }^{(24)}$. Embora as mídias sejam uma ferramenta educacional poderosa que os enfermeiros e outros profissionais de saúde podem mobilizar para disseminar informações e influenciar o comportamento do público, se usadas de forma inadequada, podem ser simultaneamente uma fonte de informações enganosas. Estudo realizado por Li e colaboradores ${ }^{(25)}$, examinando 69 vídeos relacionados à epidemia pelo COVID-19 (totalizando 257.804.146 visualizações), mostra que $27,4 \%$ dos vídeos mais vistos no YouTube continham informações enganosas ou erradas, que foram objeto de mais de 62 milhões de visualizações em todo o mundo.

Por fim, algumas limitações do presente estudo merecem ser mencionadas. Dentre elas, destaca-se o curto recorte temporal do levantamento realizado vis-à-vis à rápida produção e atualização de informações sobre a doença, processo dinâmico e de caráter quase contínuo. O objetivo era traçar um retrato, de caráter exploratório e necessariamente restrito, de como essas atividades vem sendo desenvolvidas nos NT estaduais do Programa Telessaúde Brasil Redes. Ressalta-se, contudo, que novos conteúdos e outras iniciativas que visam promover a saúde em tempos de pandemia podem ter sido disponibilizadas nos NT posteriormente à coleta de dados para este manuscrito. A falta de link ativo ou a exigência de procedimento mediado por senha para o acesso não permitiu verificar o objeto de interesse em questão em três Núcleos (Tocantins, Paraná e Paraíba). Além disso, a pesquisa restringiu-se aos NT estaduais; iniciativas de informação em saúde e atividades educativas relacionadas à COVID-19 não foram pesquisadas em Núcleos municipais e privados, nem junto a outros atores institucionais da área da Telessaúde, como a RUTE e a Universidade Aberta do SUS. Assim, os resultados apresentados não representam a totalidade de informações e atividades com potencial de estarem sendo produzidas e veiculadas usando essa modalidade de tele-educação no curso da pandemia. Por fim, a grande maioria das páginas dos NT não apresenta registro visível do número de acessos, ou do tipo de público (profissionais de saúde, gestores, cidadãos) que busca as informações, aspecto certamente interessante de ser objeto de análise.

Para além das limitações, enfatiza-se que todas as iniciativas produzidas pelos NT em tempos de pandemia reforçam a necessidade de se assegurar conectividade e infraestrutura, como forma de otimização das informações e conteúdos educativos disponibilizados. Sem conectividade e infraestrutura, a simples presença nos Núcleos não assegura acesso da população à informação em saúde ou a articulação entre os serviços, enfermeiros e outros profissionais de saúde, importantes sempre e, ainda mais, nesses tempos de pandemia.

\section{CONSIDERAÇÕES FINAIS}

A produção de informação segura, de forma ágil e transparente, visando a instrumentalizar profissionais de saúde e fortalecer a autonomia da 
população para o autocuidado, é uma iniciativa relevante que os Núcleos de Telessaúde, no Brasil, vêm desenvolvendo para o enfrentamento da pandemia da COVID-19. Observou-se um significativo esforço dos Núcleos de Telessaúde para produzir e divulgar conhecimentos e materiais, dentre outros recursos de informação e educação em saúde sobre a COVID-19, que são essenciais para atuar frente a um contexto de muitas dúvidas e incertezas sobre a doença. Desse modo, as iniciativas produzidas pelos NT evidenciam uma tentativa de viabilizar o acesso de enfermeiros e profissionais de saúde a tal produção, que certamente somam-se a outros esforços para a qualificação desses trabalhadores.

O cenário da pandemia - cuja duração ainda é desconhecida, mas se prevê longa - exige estratégias contínuas e empenho de todos. Durante a pesquisa, verificou-se que a utilização das TIC para produção, divulgação e democratização de informação e educação em saúde relacionada a essa temática vem se consolidando e segue, avançando com o auxílio de ferramentas digitais. Identificou-se que as iniciativas operadas pelos Núcleos nos diferentes territórios do país podem conferir maior visibilidade e promover a difusão das ações de promoção da saúde e de capacitação profissional, as quais podem ser replicadas e/ou readaptadas conforme as necessidades locais.

Considerando tal potencialidade, espera-se que as informações em saúde mediadas pelas TIC estimulem e fortaleçam o uso da Telessaúde, no Brasil, e sejam fonte de estudos e de iniciativas no pós-pandemia, para atender à demanda de profissionais de saúde e população.

\section{REFERÊNCIAS}

1- Wolrd Health Organization (WHO). DirectorGeneral's opening remarks at the media briefing on COVID-19. Genebr: WHO; 2020 [cited 2020 Jun 16].

Available in:

https://www.who.int/dg/speeches/detail/who-

director-general-s-opening-remarks-at-themedia-briefing-on-covid-19---11-march-2020

2- World Health Organization (WHO). Coronavirus disease 2019 (COVID-19) - Situation Report [cited 2020 Jun 20]; 148. Available in: https://www.who.int/docs/default-

source/coronaviruse/situation-

reports/20200616-covid-19-sitrep-148-

draft.pdf?sfvrsn=9b2015e9 2

3- Walker PGT, Whittaker C, Watson O, Baguelin $M$, Ainslie KEC, Bhatia $S$, et al. The global impact of
COVID-19 and strategies for mitigation and suppression. London: Imperial College London; 2020. DOI: $10.1126 /$ science.abc0035

4- Mayr V, Nußbaumer-Streit B, Gartlehner G. Quarantäne alleine oder in kombination mit weiteren public-health-maßnahmen zur eindämmung der COVID-19 pandemie: Ein cochrane rapid review. Gesundheitswesen 2020;82(6):501-6. DOI: 10.1055/a-1164-6611

5- 5.Smith AC, Thomas E, Snoswell CL, Haydon $H$, Mehrotra A, Clemensen J, et al. Telehealth for global emergencies: Implications for coronavirus disease 2019 (COVID-19). J Telemed Telecare 2020;26(5):309-13.

DOI: 10.1177/1357633X20916567

6- Brasil. Ministério da Saúde. Custeio dos Núcleos de Telessaúde: Manual instrutivo. Brasília: Ministério da Saúde; 2015.

7- Caetano R, Silva AB, Guedes ACM, Paiva CCN, Ribeiro GR, Santos DL, et al. Desafios e oportunidades para telessaúde em tempos da pandemia pela COVID-19: Uma reflexão sobre os espaços e iniciativas no contexto brasileiro. Cad Saúde Pública 2020;36(5):1-16. DOI: 10.1590/0102-311x00088920

8- Fagherazzi G, Goetzinger C, Rashid MA, Aguayo GA, Huiart L. Digital health strategies to fight COVID-19 worldwide: Challenges, recommendations, and a call for papers. J Med Internet Res. 2020;22(6):e19284. DOI: 10.2196/19284

9- Silva AB, Moraes IHS. O caso da Rede Universitária de Telemedicina: Análise da entrada da telessaúde na agenda política brasileira. Physis 2012;22(3):1211-35. DOI: 10.1590/S010373312012000300019

10- Brasil. Ministério da Saúde. Portaria no 2.546, de 27 de outubro de 2011. Redefine e amplia o Programa Telessaúde Brasil, que passa a ser denominado Programa Nacional Telessaúde Brasil Redes. Diário Oficial da União 2011.

11- Brasil. Ministério da Saúde. Programa Telessaude Brasil Redes. Núcleo de Telessaúde no Brasil. Brasília: Ministério da Saúde; 2013 [citado em 22 jun 2020]. Disponível em: https://www.saude.gov.br/telessaude/nucleosde-telessaude

12- Dolny LL, Lacerda JT, Natal S, Calvo MCM. Serviços de telessaúde como apoio à educação permanente na atenção básica à saúde: Uma proposta de modelo avaliativo. Interface 2019;23:1-18. DOI: 10.1590/interface.180184 13- Yin RK. Estudo de caso: Planejamento e métodos. 5a ed. Porto Alegre: Bookman; 2015. 
14- Brasil. Ministério da Saúde. Nota técnica 50/2015 DEGES/SGTES/MS. Diretrizes para a oferta de atividades do Programa Nacional Telessaúde Brasil Redes. Brasília: Ministério da Saúde; 2015.

15- Brasil. Portaria MS no 467, de 20 de março de 2020. Dispõe, em caráter excepcional e temporário, sobre as ações de Telemedicina, com o objetivo de regulamentar e operacionalizar as medidas de enfrentamento da emergência de saúde pública de importância internacional previstas no art. 3ㅇ da Lei no 13.979, de 6 de fevereiro de 2020, decorrente da epidemia de COVID-19. Diário Oficial da União 2020.

16- Brasil. Ministério da Saúde. Plano de contingência nacional para infecção humana pelo novo Coronavírus Covid-19. Brasília: Centro de Operações de Emergências em Saúde Pública; 2020 [citado em 30 mar 2020]. Disponível em: https://portalarquivos2.saude.gov.br/images/pdf 2020/fevereiro/13/plano-contingenciacoronavirus-COVID19.pdf

17- Corrêa EJ, Araújo MRN, Lima MCPB, Cadete MMM, Bonolo $P$, Cristófaro MAC, et al. Universidade Aberta do Sistema Único de Saúde: A participação da Universidade Federal de Minas Gerais de 2007 a 2017. In: Campos FE, Lemos AF, Vianna RF, Oliveira VA, Franco SM, Nascimento $E M$, et al., organizadores. Experiências exitosas da Rede UNA-SUS: Trajetórias de fortalecimento e consolidação da educação permanente em saúde no Brasil. São Luís: EDUFMA; 2017. p. 231-48.

18- França T, Rabello ET, Magnago C. As mídias e as plataformas digitais no campo da Educação Permanente em Saúde: Debates e propostas. Saúde Debate 2019;43(nesp 1):106-15. DOI: 10.1590/0103-11042019s109

19- Sardi L, Idri A, Fernández-Alemán JL. A systematic review of gamification in e-Health. J Biomed Inform. 2017; 71:31-48. DOI: 10.1016/i.jbi.2017.05.011

20- Fundação Owsaldo Cruz (FIOCRUZ). Livretos de atividades para crianças na quarentena. Rio de Janeiro: Fiocruz; 2020 [citado em 17 jun 2020]. Disponível em: https://portal.fiocruz.br/coronavirus/materialpara-download

21- Greenhalgh T, Koh GCH, Car J. Covid-19: Avaliação remota em Atenção Primária à Saúde. Rev Bras Med Fam Comunidade 2020;15(42):2461. DOI: 10.5712/rbmfc15(2)2461 22- Ladaga FMA, Andrade G, Sartori A, Yamaguchi MU. WhatsApp, uma ferramenta emergente para a promoção da saúde.
Enciclopédia Biosfera 2018; 15(28):1370-84. DOI: 10.18677/EnciBio 2018B107

23- Lazer DMJ, Baum MA, Benkler Y, Berinsky AJ, Greenhill KM, Menczer F, et al. The science of fake news. science. 2018;359(6380):1094-6. DOI: 10.1126/science.aao2998

24- Basch CH, Hillyer GC, Meleo-Erwin ZC, Jaime C, Mohlman J, Basch CE. Preventive behaviors conveyed on youtube to mitigate transmission of COVID-19: Cross-sectional study. JMIR Public Health Surveill 2020; 6(2):e18807. DOI: $10.2196 / 18807$

25- Li HO, Bailey A, Huynh D, Chan J. YouTube as a source of information on COVID-19: A pandemic of misinformation? BMJ Glob Health 2020;5(5):e002604. DOI: 10.2139/ssrn.3569884

Nota: O artigo foi apoiado pelo Conselho Nacional de Desenvolvimento Científico e Tecnológico (CNPq), no processo 305439/2017-4.

Recebido em: 04/07/2020

Aprovado em: 24/09/2020

Endereço de correspondência:

Rosângela Caetano

Instituto de Medicina Social-UERJ. Rua São Francisco Xavier, 524, 70 andar, blocos D e F. Maracanã, Rio de Janeiro-RJ, Brasil. CEP 20550-013

E-mail: caetano.r@gmail.com 